\title{
Neuronal coding in the rodent prefrontal cortex
}

\author{
Olga Kornienko ${ }^{1 *}$, Liya Ma², James M Hyman², Jeremy K Seamans², Daniel Durstewitz ${ }^{1}$ \\ From Twenty Second Annual Computational Neuroscience Meeting: CNS*2013 \\ Paris, France. 13-18 July 2013
}

Apart from being associated with working memory, neurons in the rodent medial prefrontal cortex (mPFC) are known to be involved in encoding of spatial and temporal contexts [1], the deduction of rules [2], and decision making [3]. The context-dependent organization of neural assemblies encoding for different task events, stimuli or decisions $[3,4]$, may account for the great flexibility required during the performance of higher cognitive tasks. The way in which single neurons and their interactions code for different entities may play a huge role in this flexibility [5], but has rarely been systematically investigated in the PFC.

Here, we employ various multivariate statistical techniques and time series bootstraps to analyze the way in which neurons, neural interactions, and temporal patterns of activity within ensembles of simultaneously recorded rat PFC neurons contribute to the neural population code during the performance of different tasks comprised of multiple stimuli, task events, and responses.

To examine the neural population representation of a given set of stimuli and task events, in a first step kernel density stimates of spiking activity were obtained from all recorded neurons. Both multivariate/ multiple regression and classification approaches were then utilized to characterize neuronal coding properties. Using regression, the distributions of single neuron contributions to the explained variation in stimulus conditions were charted, both individually and after regressing out or taking into account the contribution of other neurons. The same was done including neuronal interaction terms of various orders as well as time-lagged versions of the neuronal activities (based on the idea of delay-embedding, thus taking temporal patterns into account). Significant contributions of single terms or sets of terms were identified by construction of null hypothesis distributions through

\footnotetext{
* Correspondence: olga.kornienko@zi-mannheim.de

'Bernstein Center for Computational Neuroscience, Central Institute of Mental Health, Medical Faculty Mannheim, Heidelberg University, 68159, Germany

Full list of author information is available at the end of the article
}

block-permutation bootstraps. In a complementary decoding-type of analysis, a linear discriminant analysis (LDA) classifier was run on sets of single neuron activities, timelagged versions of these, and their interaction terms, with performance evaluated through leave-one-out cross-validation. Results show that 1) contributions to explained variation in stimulus conditions follow monotonically falling, potentially power-law-like, distributions, and 2) both including temporal pattern information as well as neural interaction terms significantly improves prediction performance and strongly reduces the misclassification rate.

These findings indicate that a) there appears to be no highly specialized subpopulation of neurons encoding for specific events, and b) that precise temporal patterns, and to a lesser degree correlations among units, have a major contribution to the neural representation of specific stimuli and internal task stages in the rat mPFC.

\section{Acknowledgements}

This work was funded by grants from the German ministry for education and research (BMBF, 01GQ1003B) and the Deutsche Forschungsgemeinschaft to D.D. (Du 354/7-2, SFB-636 B08).

\section{Author details}

${ }^{1}$ Bernstein Center for Computational Neuroscience, Central Institute of Mental Health, Medical Faculty Mannheim, Heidelberg University, 68159, Germany. ${ }^{2}$ Brain Research Centre, Psychiatry, Faculty of Medicine, University of British Columbia, Vancouver, BC, BC V6T 2B5, Canada.

Published: 8 July 2013

\section{References}

1. Hyman JM, Ma L, Balaguer-Ballerster E, Durstewitz D, Seamans JK: Contextual encoding by ensembles of medial prefrontal cortex neurons. PNAS 2012, 109:5086-5091.

2. Durstewitz D, Vittoz NM, Floresco SB, Seamans JK: Abrupt transitions between prefrontal neural ensemble states accompany behavioral transitions during rule learning. Neuron 2010, 66:334-336.

3. Lapish CC, Durstewitz D, Chandler LJ, Seamans JK: Successful choice behavior is associated with distinct and coherent network states in anterior cingulate cortex. PNAS 2008, 105:11963-11968.

4. Balaguer-Ballerster E, Lapish CC, Seamans JK, Durstewitz D: Attracting Dynamics of Frontal Cortex Ensembles during Memory-Guided DecisionMaking. PLoS Comput Biol 2011, 7:e1002057.
C Biomed Central

C 2013 Kornienko et al; licensee BioMed Central Ltd. This is an Open Access article distributed under the terms of the Creative Commons Attribution License (http://creativecommons.org/licenses/by/2.0), which permits unrestricted use, distribution, and reproduction in any medium, provided the original work is properly cited. 
5. Rigotti M, Ben Dayan Rubin D, Wang XJ, Fusi S: Internal representation of task rules by recurrent dynamics: the importance of the diversity of neural responses. Front Comput Neurosci 2010, 4:24.

doi:10.1186/1471-2202-14-S1-P117

Cite this article as: Kornienko et al:: Neuronal coding in the rodent prefrontal cortex. BMC Neuroscience 2013 14(Suppl 1):P117.

Submit your next manuscript to BioMed Central and take full advantage of:

- Convenient online submission

- Thorough peer review

- No space constraints or color figure charges

- Immediate publication on acceptance

- Inclusion in PubMed, CAS, Scopus and Google Scholar

- Research which is freely available for redistribution

Submit your manuscript at www.biomedcentral.com/submit

() BioMed Central 